\title{
Preconception Care Health Education for Female Workers of Reproductive Age in Japan Evaluation up to 6 Months after the Program
}

\author{
Midori Nagusa' ${ }^{1,2}$, Ayako Sasaki ${ }^{3}$ \\ ${ }^{1}$ Graduate School of Nursing, Osaka Medical College, Osaka, Japan \\ ${ }^{2}$ Faculty of Nursing, Setsunan University, Osaka, Japan \\ ${ }^{3}$ Faculty of Nursing, Osaka Medical College, Osaka, Japan \\ Email:m-nagusa@nrs.setsunan.ac.jp, ayabe@osaka-med.ac.jp
}

How to cite this paper: Nagusa, M. and Sasaki, A. (2019) Preconception Care Health Education for Female Workers of Reproductive Age in Japan Evaluation up to 6 Months after the Program. Health, 11, 1373-1395. https://doi.org/10.4236/health.2019.1110106

Received: September 14, 2019

Accepted: October 19, 2019

Published: October 22, 2019

Copyright $\odot 2019$ by author(s) and Scientific Research Publishing Inc. This work is licensed under the Creative Commons Attribution International License (CC BY 4.0).

http://creativecommons.org/licenses/by/4.0/

c) (i) Open Access

\begin{abstract}
Objectives: The aim of this study was to implement a health education program for education of preconception care (PCC) for female workers of reproductive age through a seminar and to evaluate the program based on PCC knowledge and awareness and behaviors before the seminar and at 3 and 6 months after the seminar. Methods: A small group health education seminar was implemented using leaflets for 84 female workers of reproductive age, age 20 to 35 years old, and an intervention by group discussion and feedback was provided. Of these participants, $60(71 \%)$ who provided valid survey responses were included in the analysis. The primary outcome indicators were PCC knowledge, PCC awareness and behaviors, and a food frequency questionnaire, and the secondary outcome indicators were self-efficacy and the health awareness and stress management subscales of the Health-Promotion Lifestyle Profile scale. The survey was conducted three times, before the seminar and at 3 and 6 months after the seminar, and the results were compared. Results: The percentage of individuals with PCC knowledge was significantly increased at 3 months after the seminar as compared with before the seminar, and it remained at this level even at 6 months after the seminar. The percentage of individuals with PCC awareness and behaviors was significantly increased for the items actively eat foods containing folic acid, prevent sexually transmitted diseases, and conduct self-examinations for breast cancer at 3 months after the seminar and for the items eat well-balanced meals, make the mind active, prevent sexually transmitted diseases, and conduct self-examinations for breast cancer at 6 months after the seminar. Furthermore, the score for the health awareness subscale of the Health-Promotion Lifestyle Profile scale was significantly increased at 6 months after the seminar.
\end{abstract}


Conclusion: The health education program established PCC knowledge at 6 months after the seminar and improved some PCC awareness and behaviors. Therefore, it was suggested that the program would be effective for education of PCC for female workers of reproductive age.

\section{Keywords}

Preconception Care, Reproductive Age, Female Workers, Health Education Program, FFQg

\section{Introduction}

Preconception care (PCC) refers to care for women and couples to manage their health for future pregnancies and improve their health [1]. PCC was proposed in Europe and the United States to further improve the prognosis of mothers and children [2]. Particularly in recent years, attention has been focused on the effectiveness of PCC for risk factors of pregnancy outcomes because of evidence presented in the guidelines recommended by the US Centers for Disease Control Prevention (CDC, 2006) [3]. Overseas, the US CDC (2006) and the World Health Organization (WHO, 2012) have already made full-fledged recommendations [1].

In Japan, education about sex and lifelong health knowledge is provided in elementary, junior high, and high schools [4] [5], and in addition to school education, a family planning consultation services are provided as premarital education services based on the Maternal and Child Health Act; however, there is no system for proper pregnancy and childbirth education before pregnancy. In recent years, the average age of women at first marriage has risen, rising to 28.8 years old in 2010 and 29.4 years old in 2015 [6]. It has been pointed out that late marriage in women is associated with infertility due to reduced fertility [7]; in fact, $18.2 \%$ of couples have been tested or treated for infertility (or are currently doing so), and this value is $28.2 \%$ in couples with no children [8]. In addition, the number of low-birth-weight infants due to excessive underweight in women is increasing year by year [6]. Due to malnutrition during the fetal period, low-birth-weight infants face the problem of $\mathrm{DOHaD}$ (developmental origins of health and disease), which increases the risk of developing lifestyle-related diseases after adulthood [9].

Therefore, in order to disseminate PCC in Japan, the Preconception Care Center was established at the National Center for Child Health and Development in 2015, and a PCC checklist tailored to the actual situation in Japan was created [1]. The contents of the checklist include no smoking, refraining from drinking, eating well-balanced meals, maintaining proper weight, exercise, reducing stress, and checking for lifestyle-related diseases, which represent general health education-related content, as well as PCC items such as intake of folic acid, prevention of rubella and sexually transmitted diseases, vaccination against rubella, breast 
cancer and cervical cancer screening, and thinking about life planning. Although PCC is provided by doctors for women with disease such as diabetes and collagen disease, which affect fertility at present, PCC educational activities are not well provided for the majority of general females of reproductive age. Therefore, information and support need to be provided in the future.

PCC educational activities are mainly needed for women who have reached puberty, adolescence, and maturity. Focusing on women who have reached maturity, the labor force participation rate by age group is $81.7 \%$ for women 25 to 29 years old and $73.2 \%$ for women 30 to 34 years old [10], and $70 \%$ of women who have reached maturity are workers. Furthermore, the working hours of $56.6 \%$ of female workers in their $20 \mathrm{~s}$ and $30 \mathrm{~s}$ exceed the legal working hours (40 hours per week), and there are reports that the breakfast skipping rate increases with working hours and that a shortage of total daily energy intake contributes to leanness [11]. The period around 30 years old, when women are moving towards their next life events, including pregnancy and childbirth, is considered the most important target of PCC. Currently, however, PCC information is not provided even during regular check-ups in the workplace, and immediate support is needed.

In this study, therefore, a PCC health education program was implemented to raise the awareness of PCC in female workers of reproductive age using the Trans-theoretical Model [12] [13] as a reference, and PCC knowledge and awareness and behaviors were compared between before and 3 and 6 months after implementing the health education program with the aim of evaluating it. We believe that this study will provide basic data for PCC educational methods to expand the application of PCC to general females of reproductive age.

\section{Study Methods}

\subsection{Definition of Terms}

In this study, PCC was defined as getting healthy support before pregnancy and developing healthy lifestyle habits to reduce risk factors for conception and pregnancy outcomes.

\subsection{Study Design}

The study was a single arm longitudinal intervention study.

\subsection{Survey Period}

The survey was performed from July 2018 to July 2019.

\subsection{Study Subjects}

Inclusion criteria were mature female workers aged 20 - 35 living in the Kinki and Tokyo metropolitan areas.

Exclusion criteria were those who were pregnant, doctors, dentists, pharmacists, public health nurses, midwives, nurses, and nutritionists. 
The reason for the exclusion is that there is a possibility of knowing about PCC at maternity checkups and vocational training schools.

\subsection{Data Collection Methods}

\section{1) Overview of the PCC health education program (Figure 1)}

The specific goals of this program were for a) female workers of reproductive age to learn about PCC and b) female workers of reproductive age to be able to act based on PCC knowledge.

Because this was a study on a health education program aimed at behavior change, the study was planned with reference to the Trans-theoretical Model, which indicate that there is a transformation process effective for 6 months depending on the stage of behavior change [12] [13]. These stages consist of the pre contemplation stage, contemplation stage, preparation stage, implementation stage, and maintenance stage, and these are models that promote behavior change through approaches according to the stages [12] [13]. It is predicted that the levels of health of female workers of reproductive age would be high because of their age; however, it is not possible to predict the stages to which all of the subjects belong. Therefore, whether the subjects were interested in preconception care was asked and confirmed with a question, "Are you interested in preconception care?" in the questionnaire conducted before the seminar. Because a previous study reported that women in Japan lack appropriate knowledge about a healthy pregnancy and childbirth [14], it was presumed that the majority of the subjects were in the pre contemplation stage and contemplation stage periods. In this study, a health education seminar, group discussion, and feedback comments were included in the intervention to provide support for female workers

Before PCC Health Education Seminar

After seminar

1 week later 3 months later 6 months later

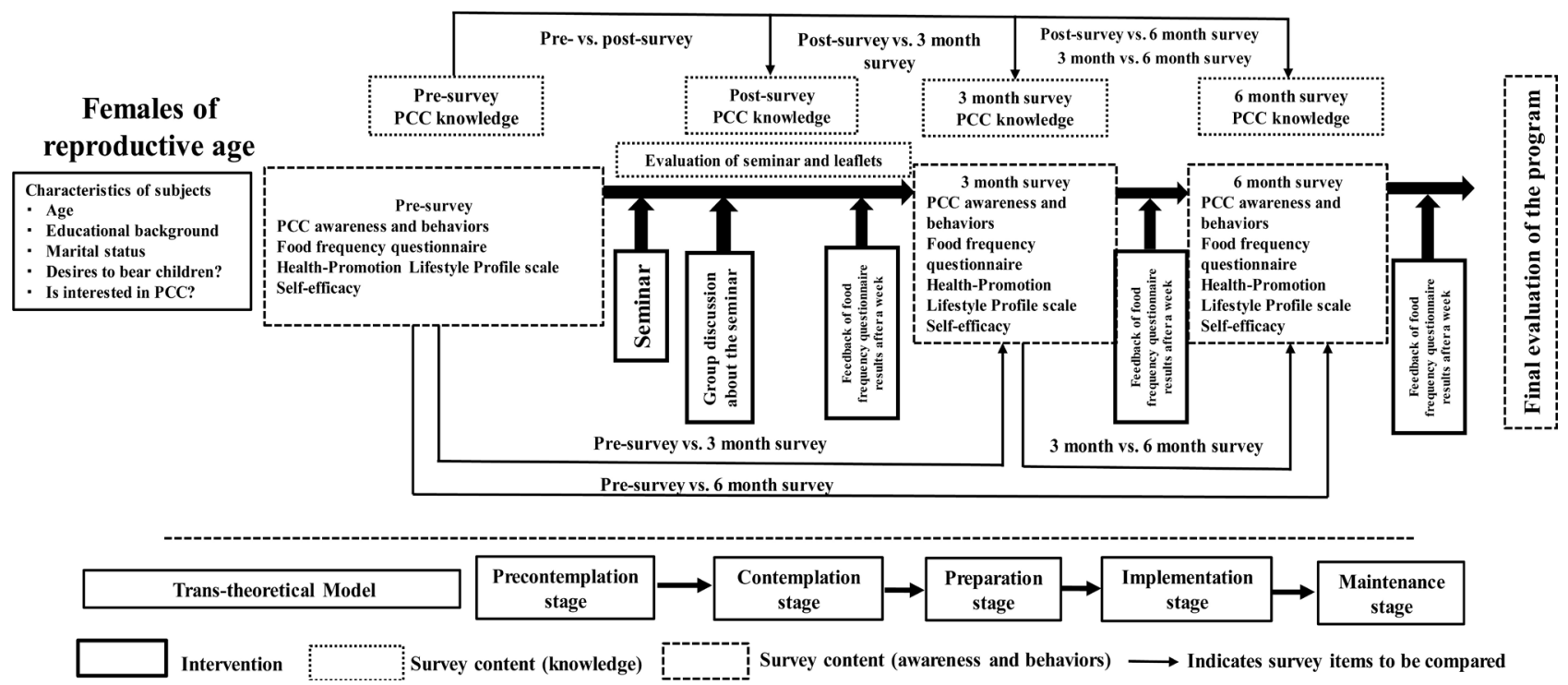

Figure 1. Research framework for evaluating preconception care health education programs for female workers of reproductive age. 
of reproductive age to take PCC action. More specifically, the intervention included the following: i) a PCC seminar; ii) a group discussion after the seminar; iii) after one week, feedback comments on the action items on the PCC checklist using the results of a pre-seminar food frequency questionnaire; and iv) after 3 months, the halfway point, feedback comments on the action items on the PCC checklist using the results of a 3-month post-seminar food frequency questionnaire. For subjects who were in the pre contemplation stage, the effects of PCC health education were explained, and encouragement was provided. For subjects who were in the contemplation stage, awareness was encouraged by providing seminar information, and time was set aside for the emotional experience of watching a video about congenital rubella syndrome. Furthermore, an opportunity for self-reevaluation was provided by the group discussion and by providing the results of food frequency questionnaires as feedback.

\section{2) Survey procedures}

Before health education

a) The subjects were asked to meet at a designated location outside of working hours and to complete a self-administered questionnaire survey (with reference number) and food frequency questionnaire (with reference number; Food Frequency Questionnaire Based on Food Groups, FFQg) [15].

PCC health education seminar (hereinafter referred to as the "seminar")

b) A seminar of about 90 minutes was held for a small group of 3 to 6 subjects using PCC leaflets created based on a literature survey. The contents of the leaflets used were created based on the results of the literature survey [16] and comprised mainly the PCC content that is lacking in Japan (Table 1). The opinions of a doctor who promotes PCC were obtained with respect to the contents of the seminar leaflets to ensure their validity.

Immediately after the seminar

c) Immediately after the seminar, a group discussion about the self-administered questionnaire survey (with reference number), leaflets, and lecture on PCC knowledge was held, and the questionnaire survey was carried out. The contents of the group discussion included the following: i) What impressed you at this

Table 1. PCC seminar content.

\begin{tabular}{cc}
\hline Item & Time allocation \\
\hline What is preconception care? & 5 minutes \\
Oocyte aging and infertility & 5 minutes \\
Life planning & 5 minutes \\
Underweight & 10 minutes \\
Folic acid & 10 minutes \\
Rubella vaccination (video viewing) \\
Sexually transmitted diseases, cervical cancer screening, \\
breast cancer self-examination \\
Total & 10 minutes \\
\hline
\end{tabular}


seminar and why; ii) what did you want to do right away with the contents of the seminar and why; and iii) which contents of the seminar did you feel were necessary and why?

d) A questionnaire survey on the ease of understanding the seminar and leaflets was carried out.

One week later

e) Short comments (100 - 200 characters) regarding PCC (energy, nutrient intake, amount of exercise, folic acid intake, vaccination against rubella, cervical cancer screening status, and criteria were evaluated) based on the individual's questionnaire survey, food frequency questionnaire results, and physical activity results were mailed as feedback within one week.

Three months later

f) At 3 months after the seminar, the self-administered questionnaire survey (with reference number) and food frequency questionnaire (with reference number) were mailed to the subjects, and the subjects were asked to return the questionnaire after completing it.

g) Short comments (100 - 200 characters) regarding PCC (energy, nutrient intake, amount of activity, folic acid intake, vaccination against rubella, cervical cancer screening status, and other criteria were evaluated) based on the individual's questionnaire survey, food frequency questionnaire results, and physical activity results were mailed as feedback within one week after return.

Six months later

h) At 6 months after the seminar, the self-administered questionnaire survey (with reference number) and food frequency questionnaire (with reference number) were mailed to the subjects, and the subjects were asked to return the questionnaire after completing it.

i) Short comments (100 - 200 characters) regarding PCC (energy, nutrient intake, amount of activity, folic acid intake, vaccination against rubella, cervical cancer screening status, and other criteria were evaluated) based on the individual's questionnaire survey, food frequency questionnaire results, and physical activity results were mailed as feedback within a week after return.

j) As the final evaluation of the program, a questionnaire survey including space for free descriptions of opinions and impressions of the entire program was mailed to the subjects, and the subjects were asked to return the questionnaire after completing it.

\subsection{Data Collected (Table 2)}

\section{1) Background of subjects}

Before the health education, the subjects were asked to provide basic information (age, height, weight, educational background), information related to PCC (marital status, desire to bear children in the future, presence or absence of interest in PCC), information related to working environment (type of occupation, employment status, working hours, place of work), and information related to 
Table 2. Survey content.

\begin{tabular}{|c|c|c|}
\hline & Item & Outcome type \\
\hline 1. Background of subjects & $\begin{array}{l}\text { Age, height, weight, marital status, family structure, desire to bear children } \\
\text { in the future } \\
\text { Educational background, type of occupation, occupation, employment } \\
\text { status, working hours, menstruation status, sleeping hours, breakfast } \\
\text { consumption, excretion status }\end{array}$ & $\begin{array}{l}\text { Characteristics of } \\
\text { subjects }\end{array}$ \\
\hline $\begin{array}{l}\text { 2. PCC knowledge and PCC awareness } \\
\text { and behaviors }\end{array}$ & $\begin{array}{l}\text { PCC knowledge: } 9 \text { items, presence or absence of knowledge } \\
\text { PCC behaviors (created with reference to the PCC checklist): } 14 \text { items, } \\
\text { presence or absence of awareness and behaviors } \\
\text { Food Frequency Questionnaire (FFQg): } 7 \text { items for daily physical activity } \\
\text { survey, } 4 \text { items for exercise time per week by entering numbers, } 29 \text { items } \\
\text { for food intake amount/food intake frequency by indicating answers with } \\
\text { numbers or a Likert scale }\end{array}$ & Primary outcome \\
\hline 3. Self-efficacy & Self-efficacy scale: 23 items, 5 -point Likert scale & Secondary outcome \\
\hline 4. Health-Promotion Lifestyle Profile & Health-Promotion Lifestyle Profile scale: 18 items, 4-point Likert scale & Secondary outcome \\
\hline $\begin{array}{l}\text { 5. Ease of understanding the seminar } \\
\text { and leaflets }\end{array}$ & $\begin{array}{l}4 \text { items, } 4 \text {-point Likert scale } \\
\text { Free descriptions of opinions and impressions }\end{array}$ & Program evaluation \\
\hline 6. Final evaluation of the program & $\begin{array}{l}1 \text { item, 3-point Likert scale; } 3 \text { items, } 4 \text {-point Likert scale } \\
\text { Free descriptions of opinions and impressions of the entire program }\end{array}$ & Program evaluation \\
\hline
\end{tabular}

physical conditions (breakfast consumption, excretion status, menstrual status, sleep time).

\section{2) PCC knowledge and PCC awareness and behaviors}

Before the health education and at 3 and 6 months after it, the subjects were asked to provide answers for 9 items regarding the presence or absence of PCC knowledge. They were also asked to provide answers for 14 items regarding the presence or absence of PCC awareness and behaviors.

\section{3) Food frequency questionnaire}

Before the health education and at 3 and 6 months after it, the subjects were asked to provide answers for 7 items of a daily physical activity survey, 4 items about exercise time per week by indicating answers numerically, and 29 items about food intake amount and frequency by indicating answers numerically or using a Likert scale. The food frequency questionnaire was a survey created with the aim of knowing the intake of specific foods and nutrients. The FFQg Excel Eiyo-kun-Food frequency questionnaire [15] used in this study is a method of estimating food intake, nutrient intake, and physical activity level based on food intake, intake frequency, and physical activity time in one-week units in the last 1 to 2 months. This survey method is able to evaluate the contents of daily meals with simple questions organized according to 29 food groups divided by food group and 10 cooking methods, and its reliability and validity have been verified.

\section{4) Self-efficacy scale}

Before the health education and at 3 and 6 months after it, the subjects were asked to complete a self-efficacy scale (23 items, 5-point Likert scale). A self-efficacy scale was used because self-efficacy reflects confidence in the ability to achieve a 
goal and is a mediator that changes behaviors. The scale used in this study was a translated version of the Self-Efficacy Scale [17], created by Sherer, and it is a scale with reliability and validity [18].

\section{5) Health-Promotion Lifestyle Profile scale}

Before the health education and at 3 and 6 months after it, the subjects were asked to complete a Health-Promotion Lifestyle Profile scale (18 items, 4-point Likert scale). The Health-Promotion Lifestyle Profile scale (Japanese version) was a translated version of the Health-Promotion Lifestyle Profile II created by Walker [19], and it is a scale with reliability and validity. Because lifestyle represents all the behaviors of individuals and includes behaviors that affect human health risks, the health awareness and stress management subscales were investigated [20].

\subsection{Seminar Content}

The PCC health education content included underweight and folic acid, which were found to be not well recognized in a literature review [16], and stress management, sexually transmitted diseases, cervical cancer screening, breast cancer self-examination, life planning, and vaccination against rubella were also added.

The following items were explained using leaflets: i) the definition of PCC, the necessity of conducting this seminar at this time [21], and about diet, exercise, state of mind, and lifestyle; ii) about the current state of oocyte aging and infertility and about considering life planning [22]; iii) about the fact that excessive underweight increases the probability of giving birth to a low-birth-weight infant and about impacts of excessive underweight on child health [21]; iv) about the fact that folic acid deficiency during pregnancy leads to fetal neural tube closure disorders and causes very serious disorders such as anencephaly and spina bifida [23] and about the introduction of foods rich in folic acid; v) about the fact that sexually transmitted diseases cause infertility, about the fact that rubella infection in early pregnancy causes fetal rubella syndrome, about how to prevent rubella [24], and a video that was viewed (Stop rubella!-Do you know about congenital rubella syndrome?) [25]; and vi) a presentation about cervical cancer, which is increasing rapidly among women in their $20 \mathrm{~s}$, and an explanation about screening for prevention [21]. The explanation about this screening was given while demonstrating self-examination for breast cancer.

\subsection{Research Question}

When we carry out a health education program for preconception care (PCC) awareness for female workers of reproductive age, we have knowledge about PCC three months and six months later than before the seminar. More people act with awareness.

\subsection{Study Hypotheses}

1) The percentages of PCC knowledge and PCC awareness and behaviors and values for nutrient intake are significantly higher at 3 and 6 months after the 
health education seminar than before the seminar.

2) The scores for self-efficacy and the health awareness and stress management subscales of the Health-Promotion Lifestyle Profile scale are significantly higher at 3 and 6 months after the health education seminar than before the seminar.

\subsection{Analysis Methods}

1) The survey items were analyzed by descriptive statistics. Cochran's $Q$ test and Bonferroni's multiple comparison test were used to compare PCC knowledge and to compare PCC awareness and behaviors between before the PCC health education seminar and at 3 and 6 months after the seminar. Furthermore, for comparison of nutrient intake according to the food frequency questionnaire, self-efficacy, and the health awareness and stress management subscales of the Health-Promotion Lifestyle Profile scale before the PCC health education seminar and at 3 months after the seminar, the Friedman test and Bonferroni's multiple comparison test were used. The significance level was less than 5\%. SPSS Statistics Ver. 25 was used as the statistical software.

2) The contents of the group discussion were coded. For opinions and impressions about the seminar and leaflets and free descriptions of opinions and impressions about the entire program, common codes were categorized. Data were examined for validity of naming and analyzed a by both researchers.

\subsection{Ethical Considerations}

This study was performed after obtaining approval from the Research Ethics Committee of Osaka Medical College (Approval number: KAN-95 [2465]) as well as the Research Ethics Review Committee for Humans of Setsunan University, the university to which M.N. belongs (Approval number: 2018-032). Regarding participation in the survey, explanatory materials and an explanation of the study were provided to the subjects. These explained that research cooperation was based on the subject's free will, that doubts and objections could be raised with researchers as appropriate; that subjects could freely withdraw in the middle of the study without any disadvantages; that the results obtained from the survey would be used only for presentations at academic conferences and publication in academic papers and that when results were published, care would be taken to ensure that subjects could not be identified; and that when investigating changes in the current situation and in the future, this research data might be used secondarily for comparison. Consent to participate in the research was obtained from the subjects in the form of a signed consent form.

\section{Results}

\subsection{Recovery of Questionnaire Forms (Figure 2)}

The surveys were conducted with 84 female workers of reproductive age as participants. The recovery rate of the questionnaire forms in the surveys before and immediately after the seminar was $100 \%$. The survey at 3 months after the se- 
minar was conducted with 75 participants, and the recovery rate was $89.3 \%$. The survey at 6 months was conducted with 60 participants, and the recovery rate was $71.4 \%$. Analyses were performed for the 60 participants for which data were available up to the survey at 6 months.

\subsection{Subject Attributes (Table 3)}

Mean age, height, weight, and BMI of the 60 subjects were $28.0 \pm 4.0$ years old, $159.9 \pm 5.5 \mathrm{~cm}, 51.1 \pm 5.2 \mathrm{~kg}$, and BMI $20.0 \pm 2.1$, respectively. Marital status was showed in Figure 2. Survey flow chart married for 10 subjects (16.7\%) and unmarried for 50 subjects $(83.3 \%)$, and for desire to bear children, 51 subjects (85.0\%) desired to bear children, 8 subjects (13.3\%) did not desire to bear children, and 1 subject left the item unanswered (1.7\%). For interest in PCC, 50 subjects $(83.3 \%)$ had interest, and 10 subjects (16.7\%) had no interest. For educational background, 45 subjects $(75.0 \%)$ graduated from university or graduate school, and for employment status, 52 subjects (86.7\%) were full-time employees.

\subsection{Comparison of the Percentages of Individuals with PCC Knowledge before the Seminar and at 3 and 6 Months after the Seminar (Table 4)}

Among the 9 items related to PCC knowledge before the seminar, the items that

Table 3. Subject attributes $(n=60)$.

\begin{tabular}{|c|c|c|c|}
\hline Item & Response & Number & $\%$ \\
\hline \multirow{2}{*}{ Marriage status } & Married & 10 & 16.7 \\
\hline & Unmarried & 50 & 83.3 \\
\hline \multirow{2}{*}{ Delivery history } & Yes & 1 & 1.7 \\
\hline & No & 59 & 98.3 \\
\hline \multirow{3}{*}{ Desire to bear children } & Yes & 51 & 85.0 \\
\hline & No & 8 & 13.3 \\
\hline & Blank & 1 & 1.7 \\
\hline \multirow{2}{*}{ Interest in PCC } & Yes & 50 & 83.3 \\
\hline & No & 10 & 16.7 \\
\hline Educational & Junior high/high school & 6 & 10.0 \\
\hline \multirow{2}{*}{ Background } & Technical school, junior college, technical college & 9 & 15.0 \\
\hline & University/graduate school & 45 & 75.0 \\
\hline \multirow{2}{*}{ Employment status } & Full-time employee & 52 & 86.7 \\
\hline & Non-full-time employee & 8 & 13.3 \\
\hline \multirow{4}{*}{ Job content } & Clerical position & 31 & 51.7 \\
\hline & Sales & 5 & 8.3 \\
\hline & Others & & \\
\hline & $\begin{array}{l}\text { Elementary school teacher, junior high school } \\
\text { teacher, piano instructor, hairdresser, etc. }\end{array}$ & 23 & 38.3 \\
\hline
\end{tabular}




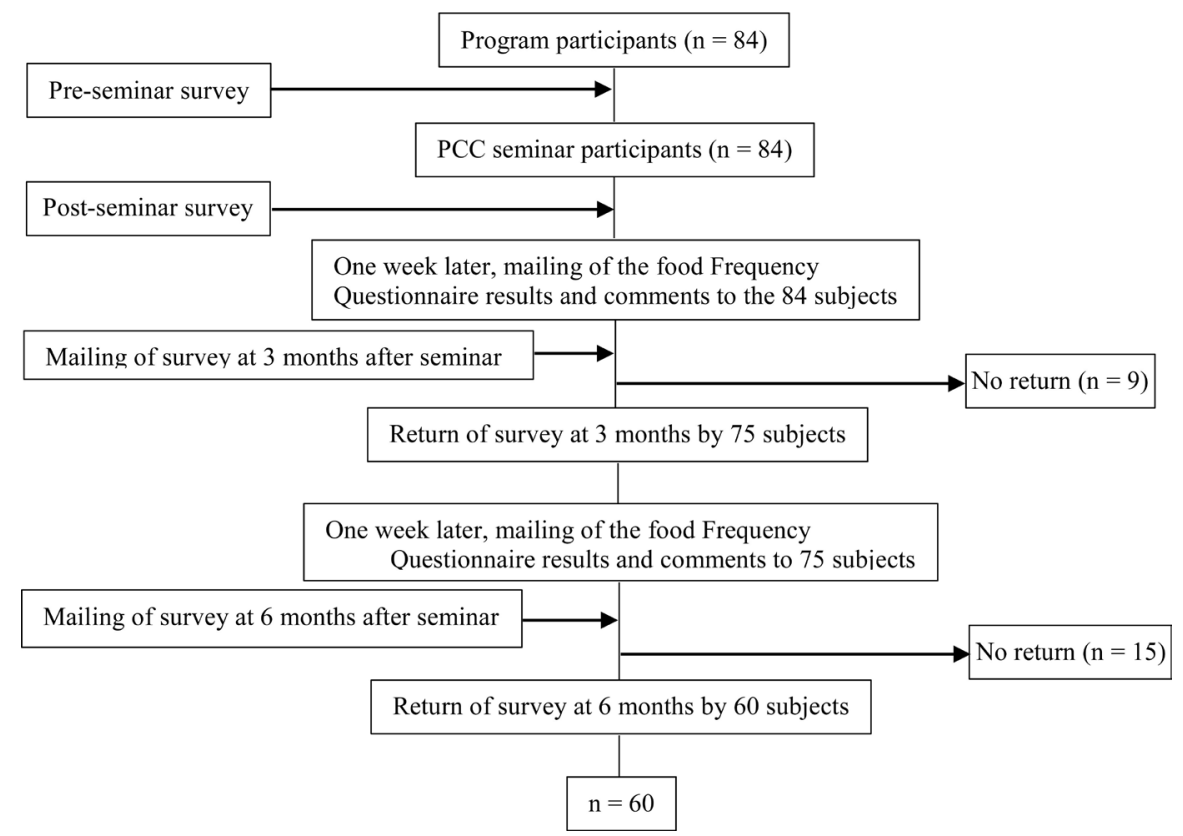

Figure 2. Survey flow chart.

Table 4. Comparison of the percentages of subjects with PCC knowledge before the seminar and at 3 and 6 months after the se$\operatorname{minar}(\mathrm{n}=60)$.

\begin{tabular}{|c|c|c|c|c|}
\hline Question content & $\begin{array}{l}\text { Before seminar } \\
\text { Number (\%) }\end{array}$ & $\begin{array}{l}\text { After } 3 \text { months } \\
\text { Number (\%) }\end{array}$ & $\begin{array}{l}\text { After } 6 \text { months } \\
\text { Number (\%) }\end{array}$ & $\begin{array}{l}\text { Significance } \\
\text { p value }\end{array}$ \\
\hline Oocyte aging & $57(95.0)$ & $59(98.3)$ & $60(100)$ & NS \\
\hline Decrease of fertility & $52(86.7)$ & $59(98.3)^{*}$ & $60(100)^{\star}$ & $\mathrm{p}<0.01$ \\
\hline Underweight and low-birth-weight infants & $20(33.3)$ & $56(93.3)^{*}$ & $59(98.3)^{*}$ & $\mathrm{p}<0.01$ \\
\hline Low-birth-weight infants and lifestyle-related diseases & $8(13.3)$ & $44(73.3)^{*}$ & $55(91.7)^{*}$ & $\mathrm{p}<0.01$ \\
\hline Folic acid and neural tube closure disorder & $11(18.3)$ & $57(95.0)^{*}$ & $60(100)^{*}$ & $\mathrm{p}<0.01$ \\
\hline Effects of rubella on the fetus & $42(70.0)$ & $60(100)^{*}$ & $60(100)^{*}$ & $\mathrm{p}<0.01$ \\
\hline Sexually transmitted diseases and infertility & $25(41.7)$ & $57(95.0)^{*}$ & $59(98.3)^{*}$ & $\mathrm{p}<0.01$ \\
\hline Cervical cancer screening from age 20 & $47(78.3)$ & $59(98.3)^{*}$ & $58(96.7)^{*}$ & $\mathrm{p}<0.01$ \\
\hline Pregnancy/childbirth and life planning & $48(80.0)$ & $48(80.0)$ & $48(80.0)$ & NS \\
\hline
\end{tabular}

Values represent the number (\%) of participants who answered "I know about it". Multiple comparison was performed between before the seminar and 3 and 6 months after the seminar with Cochran's $\mathrm{Q}$ test and the Bonferroni method. ${ }^{*} \mathrm{p}<0.01$. NS: not significant.

had more respondents who answered "I know about it" than respondents who answered "I don't know about it" were oocyte aging (57 subjects, 95.0\%), decrease of fertility with aging (52 subjects, $86.7 \%$ ), pregnancy/childbirth and life planning (48 subjects, $80.0 \%$ ), cervical cancer screening from age 20 (47 subjects, $78.3 \%)$, and rubella prevalence in pregnant women and the effects on the fetus (42 subjects, 70.0\%). On the other hand, items that had fewer respondents who answered "I know about it" than respondents who answered "I don't know about it" were low-birth-weight infants and lifestyle-related diseases (8 subjects, $13.3 \%$ ), folic acid and neural tube closure disorder (11 subjects, $18.3 \%)$, under- 
weight in pregnant women and low-birth-weight infants (20 subjects, 33.3\%), and sexually transmitted diseases and infertility ( 25 subjects, $41.7 \%$ ).

When comparing the percentages of subjects who answered "I know about it" using Cochran's $Q$ test between before and 3 and 6 months after the seminar in the 9 items related to PCC knowledge, significant differences were observed in 7 out of 9 items. Multiple comparison using the Bonferroni method revealed that awareness concerning the decrease of fertility with aging, underweight in pregnant women and low-birth-weight infants, low-birth-weight infants and lifestyle-related diseases, folic acid and neural tube closure disorder, rubella prevalence in pregnant women and the effects on the fetus, sexually transmitted diseases and infertility, and cervical cancer screening from age 20 was significantly higher at 3 and 6 months after the seminar as compared with before the seminar.

\subsection{Comparison of PCC Awareness and Behaviors before the Seminar and at 3 and 6 Months after the Seminar (Table 5)}

Among the 14 items related to PCC awareness and behaviors, the items that had more respondents who answered "I do it" than respondents who answered "I don't do it" were prevent sexually transmitted diseases (56 subjects, 93.3\%), quit smoking (56 subjects, 93.3\%), undergo medical examinations (51 subjects, $85.0 \%$ ), vaccinated against rubella (44 subjects, $73.3 \%$ ), avoid stress (39 subjects, $65.0 \%$ ),

Table 5. Comparison of the percentages of subjects with PCC awareness and behaviors before the seminar and 3 and 6 months after the seminar $(n=60)$.

\begin{tabular}{ccccc}
\hline Question content & $\begin{array}{c}\text { Before seminar } \\
\text { Number }(\%)\end{array}$ & $\begin{array}{c}\text { After 3 months } \\
\text { Number (\%) }\end{array}$ & $\begin{array}{c}\text { After } 6 \text { months } \\
\text { Number (\%) }\end{array}$ & $\begin{array}{c}\text { Significance } \\
\text { p value }\end{array}$ \\
\hline Refrain from alcohol & $41(68.3)$ & $46(76.7)$ & $45(75.0)$ & NS \\
Eat well-balanced meals & $33(55.0)$ & $34(56.7)$ & $43(71.7)^{*}$ & $\mathrm{p}<0.05$ \\
Maintain a proper weight & $35(58.3)$ & $41(68.3)$ & $39(65.0)$ & NS \\
Active intake of folic acid & $29(48.3)$ & $40(66.7)^{*}$ & $39(65.0)$ & $\mathrm{p}<0.05$ \\
Exercise actively & $29(48.3)$ & $28(46.7)$ & $31(51.7)$ & NS \\
Make the mind active & $32(53.3)$ & $38(63.3)$ & $41(68.3)^{*}$ & $\mathrm{p}<0.05$ \\
Avoid stress & $39(65.0)$ & $38(63.3)$ & $44(73.3)$ & $\mathrm{NS}$ \\
Prevent sexually transmitted diseases & $56(93.3)$ & $59(98.3)^{*}$ & $59(98.3)^{*}$ & $\mathrm{p}<0.05$ \\
Vaccinated against rubella & $44(73.3)$ & $36(60.0)$ & $39(65.0)$ & NS \\
Quit smoking & $56(93.3)$ & $56(93.3)$ & $57(95.0)$ & NS \\
Undergo medical examinations & $51(85.0)$ & $49(81.7)$ & $51(85.0)$ & NS \\
Undergo cervical cancer screening & $31(51.7)$ & $32(53.3)$ & $33(55.0)$ & NS \\
Undergo breast cancer screening & $17(28.3)$ & $15(25.0)$ & $14(23.3)$ & NS \\
Conduct self-examinations for & $3(5.0)$ & $16(26.7)^{* *}$ & $21(35.0)^{* *}$ & $\mathrm{p}<0.01$ \\
breast cancer & & & & \\
\hline
\end{tabular}

Values represent the number (\%) of participants who answered "I do it". Multiple comparison was performed between before the seminar and 3 and 6 months after the seminar with Cochran's Q test and the Bonferroni method. ${ }^{*} \mathrm{p}<0.05 ;{ }^{* *} \mathrm{p}<0.01$. NS: not significant. 
maintain a proper weight ( 35 subjects, $58.3 \%$ ), and eat well-balanced meals (33 subjects, 55.0\%). On the other hand, the items that had fewer respondents who answered "I do it" than respondents who answered "I don't do it" were conduct self-examinations for breast cancer (3 subjects, 5.0\%), undergo breast cancer screening (17 subjects, 28.3\%), undergo cervical cancer screening (31 subjects, $51.7 \%$ ), exercise actively ( 29 subjects, $48.3 \%$ ), and actively eat foods containing folic acid ( 29 subjects, $48.3 \%$ ).

When comparing the percentages of respondents who answered "I do it" between before and 3 months after the seminar, the percentages were significantly higher for actively eat foods containing folic acid, prevent sexually transmitted diseases, and conduct self-examinations for breast cancer. Furthermore, when comparing between before and 6 months after the seminar, the percentages were significantly higher for eat well-balanced meals, make the mind active, prevent sexually transmitted diseases, and conduct self-examinations for breast cancer.

\subsection{Comparison of Nutrient Intake between before the Seminar and at 3 and 6 Months after the Seminar}

Regarding the nutrient intake status before the seminar and at 3 and 6 months after the seminar, there were no significant differences in intake status for energy; protein; lipids; potassium; calcium; magnesium; phosphorus; iron; vitamins $\mathrm{D}, \mathrm{K}$, and C; folic acid; and amount of alcohol (Table 6). There were significant

Table 6. Comparison of nutrient intake of subjects before the seminar and 3 and 6 months after the seminar $(n=60)$.

\begin{tabular}{cccc}
\hline Item (unit) & Before (mean \pm SD) & 3 months (mean \pm SD) & 6 months (mean \pm SD) \\
\hline Energy $(\mathrm{kcal})$ & $1633.1 \pm 457.7$ & $1609.6 \pm 445.6$ & $1560.9 \pm 391.8$ \\
Protein $(\mathrm{g})$ & $54.8 \pm 14.9$ & $56.2 \pm 17.4$ & $55.7 \pm 15.5$ \\
Lipids $(\mathrm{g})$ & $61.4 \pm 22.2$ & $59.9 \pm 19.4$ & $59.6 \pm 17.8$ \\
Carbohydrates $(\mathrm{g})$ & $202.7 \pm 60.1$ & $199.3 \pm 59.6$ & $188.8 \pm 51.1^{* *}$ \\
Potassium $(\mathrm{mg})$ & $1683.6 \pm 556.5$ & $1723.6 \pm 561.8$ & $1688.3 \pm 571.4$ \\
Calcium $(\mathrm{mg})$ & $435.9 \pm 182.9$ & $429.1 \pm 184.9$ & $435.9 \pm 159.6$ \\
Magnesium $(\mathrm{mg})$ & $178.5 \pm 57.2$ & $181.4 \pm 55.3$ & $181.1 \pm 59.7$ \\
Phosphorus $(\mathrm{mg})$ & $789.8 \pm 229.2$ & $794.9 \pm 233.8$ & $795.5 \pm 224.5$ \\
Iron $(\mathrm{mg})$ & $6.2 \pm 2.5$ & $6.5 \pm 3.1$ & $6.4 \pm 2.2$ \\
Vitamin D $(\mu \mathrm{g})$ & $3.9 \pm 2.2$ & $4.7 \pm 3.2$ & $4.6 \pm 2.5$ \\
Vitamin K $(\mu \mathrm{g})$ & $147.6 \pm 65.0$ & $155.2 \pm 70.5$ & $158.7 \pm 80.5$ \\
Vitamin C $(\mu \mathrm{g})$ & $57.8 \pm 29.1$ & $62.0 \pm 34.0$ & $59.7 \pm 34.3$ \\
Folic acid $(\mu \mathrm{g})$ & $192.9 \pm 71.7$ & $203.2 \pm 79.5$ & $200.3 \pm 88.1$ \\
Salt equivalent $(\mathrm{g})$ & $6.2 \pm 2.0$ & $6.9 \pm 2.4$ & $7.3 \pm 3.0^{*}$ \\
Amount of alcohol $(\mathrm{g})$ & $3.9 \pm 6.1$ & $3.7 \pm 5.1$ & $3.6 \pm 4.7$ \\
\hline
\end{tabular}

Multiple comparison was performed between before the seminar and 3 and 6 months after the seminar with the Friedman test and Bonferroni method. ${ }^{*} \mathrm{p}<0.05 ;{ }^{*} \mathrm{p}<0.01$. 
differences in the intake of carbohydrates and salt equivalent.

\subsection{Comparison of the Health Awareness and Stress Management Subscales of the Health Promotion Lifestyle Profile Scale and Self-Efficacy before the Seminar and at 3 and 6 Months after the Seminar (Table 7)}

Regarding the health awareness and stress management subscales of the Health Promotion Lifestyle Profile scale and self-efficacy before the seminar and at 3 and 6 months after the seminar, the score for health awareness was significantly higher at 6 months after the seminar.

\subsection{Group Discussion}

In the group discussion, the subjects were asked for opinions about: 1) what impressed them at the seminar; 2) what they wanted to do right away with the contents of the seminar; and 3) which contents of the seminar they felt were necessary. The following is the content of the group discussion. Subject narratives are indicated with quotation marks.

1) What impressed the subjects at the seminar

a) Opinions about rubella

Two subjects said, "I was impressed by the topic of rubella," and 1 subject each said, "Is it possible to undergo a rubella antibody titer test for rubella?" "If I have rubella antibodies, do I have to take the test again?" "If I have been vaccinated against rubella, would I be okay even if my husband got rubella?" "How long do the antibodies last after vaccination against rubella?" "Where should I get an antibody titer test?" "Should my husband be vaccinated against rubella?" and "How long will the antibody titer last?"

b) Opinions about oocyte aging

One subject each said, "Is it not a problem if eggs are collected and preserved while I am young?" "Isn't it better to freeze eggs?" "Is irregular menstruation caused by aging of the ovaries?" "Shouldn't my husband lose or gain weight? Is there an effect on sperm?" "How old is the donor of the egg in the photo shown in the seminar?" and "How many people are infertile?"

c) Opinions about nutrition intake

One subject each said, "After answering the questionnaire, I realized that I

Table 7. Comparison of health awareness and stress management of the Health Promotion Lifestyle Profile scale and self-efficacy of subjects before the seminar and 3 and 6 months after the seminar $(n=60)$.

\begin{tabular}{ccccc}
\hline Item & Before & 3 months & 6 months & Significance p value \\
\hline Health awareness & 20.15 & 21.22 & $21.57^{* *}$ & $p<0.01$ \\
Stress management & 23.27 & 23.53 & 23.85 & NS \\
Self-efficacy & 73.0 & 73.53 & 72.65 & NS \\
\hline
\end{tabular}

Multiple comparison was performed between before the seminar and 3 and 6 months after the seminar with the Friedman test and Bonferroni method. ${ }^{*} \mathrm{p}<0.01$. NS: not significant 
was not eating enough," "Looking back at my meals, I noticed that I was not eating enough," "What foods contain vitamin B, and what is it good for?" "Is the content of folic acid after cooking?" and "I didn't know what was wrong with not taking folic acid".

2) What the subjects wanted to do right away with the contents of the seminar

One subject each said, "I thought I would undergo cervical cancer screening," "I want to have children, so I have to think about it," and "I thought that I should take care of my life".

3) Which contents of the seminar the subjects felt were necessary

No opinions were expressed regarding any contents of the seminar that subjects felt were necessary.

The above contents were shared among the participants during group discussions, and the researchers answered the questions.

\subsection{Evaluation of the PCC Health Education Seminar and Leaflets}

\section{1) Evaluation of the PCC health education seminar}

The subjects were asked to answer questions regarding the clarity of the seminar at the end of the seminar; all 84 subjects (100\%) answered that it was "very easy to understand" or "easy to understand," and all 84 subjects (100\%) answered that it was "very useful" or "useful".

\section{2) Evaluation of leaflets}

The subjects were asked to answer questions regarding the clarity of the contents of the leaflets at the end of the seminar; all 84 subjects (100\%) answered that they were "very easy to understand" or "easy to understand", and all 84 subjects (100\%) answered that they were "very useful" or "useful".

\section{3) Opinions and impressions about the seminar and leaflets}

Opinions and impressions about the seminar and leaflets were requested in the form of free descriptions. The responses were then coded, and common codes were categorized. As a result, 12 subcategories and 69 codes were obtained.

The subcategories and numbers of codes for each were as follows: realized the fear of rubella affecting pregnancy, 12 codes; I will get vaccinated against rubella, 3 codes; rubella antibody titer affecting babies, 1 code; I will undergo an antibody titer test for rubella, 4 codes; I want to have a checkup for cervical and breast cancer, 2 codes; I am thinking to incorporate foods containing folic acid into my diet, 4 codes; I want to keep thinking about the future, 8 codes; I was able to be aware of knowledge before becoming pregnant, 10 codes; opportunity to review my eating habits, 7 codes; easy-to-understand seminar, 14 codes; impressive video, 2 codes; and leaflets are summarized well and easy to understand, 2 codes.

\subsection{Results of the Food Frequency Questionnaire and Feedback Comments}

Based on the results for energy intake and activity in the food frequency questionnaire, subjects with insufficient energy intake were encouraged to increase their overall food intake, snacking, and energy intake. In addition, comments 
were provided to inform subjects about the sufficiency and insufficiency of each nutrient and to promote continuous intake of foods high in iron and calcium (eggs, meat, seafood, soybeans, green and yellow vegetables, fruits, seaweed) and dairy products, the intake of which tended to be insufficient. Regarding folic acid, in the case of insufficiency, the subjects were informed of the amount of insufficiency individually and encouraged to continue to eat foods high in folic acid as conveyed in the seminar. When subjects indicated that they had not been screened for cervical or breast cancer or vaccinated against rubella in the questionnaire, they were recommended to schedule it in the future, and for breast cancer self-examinations, it was commented that the subjects should develop the habit of performing a self-examination in the first week after menstruation.

\subsection{Evaluation of the PCC Health Education Program}

\section{1) Necessity of PCC health education}

At the end of the program, the subjects were asked to answer a question regarding the necessity of PCC health education, and 49 subjects (81.7\%) answered that "it is necessary".

\section{2) Desired age and place for PCC health education}

The subjects were asked to answer questions regarding the age and place at which they would like to attend PCC health education, and the most desired ages for providing the PCC health education were the early twenties (39.6\%), followed by the late teens $(10.4 \%)$, the late twenties (10.4\%), and then the early thirties (8.3\%). The most desired places for the PCC health education were university (15 subjects), followed by high school (6 subjects), workplace medical examinations ( 5 subjects), and workplace classes (5 subjects).

\section{3) Evaluation of the nutrient intake status results}

Regarding the nutrient intake status results, 55 subjects (91.6\%) answered that they were "very useful" or "useful".

\section{4) Evaluation of physical activity results}

Regarding the physical activity results, 55 subjects (91.6\%) answered that they were "very useful" or "useful".

\section{5) Evaluation of short comments}

Regarding the short comments, 59 subjects (98.3\%) answered that they were "very useful" or "useful".

6) Opinions and impressions about the entire PCC health education program

Opinions and impressions about the entire PCC health education program were requested in the form of free descriptions. The responses were then coded, and common codes were categorized. As a result, 10 subcategories and 36 codes were obtained for positive opinions, and 5 subcategories and 5 codes were obtained for problems.

The subcategories and numbers of codes for each were as follows:

a) Positive opinions

For positive opinions, the subcategories were as follows: it was an opportunity 
to review my eating habits, 16 codes; I will undergo cervical cancer screening and vaccination, 1 code; I had a rubella antibody titer test, 1 code; it is not easy to reflect in real life, 2 codes; it was an opportunity to think about lifestyle habits, 6 codes; short pieces of advice were helpful, 1 code; I was able to review information later using the leaflets, 1 code; I am glad to know about PCC, 4 codes; I wish I could have attended the program when I was a student, 1 code; and I hope this program is spread to many people, 3 codes.

b) Problems

For problems, the subcategories were as follows: the nutrition results table was difficult to understand, 1 code; the food frequency questionnaire was difficult to complete, 1 code; I wanted specific information on where I could be vaccinated, 1 code; the questionnaire takes time to complete, 1 code; and it should only be targeted at people who are interested, 1 code.

\section{Discussion}

\subsection{Actual Conditions of PCC Knowledge and PCC Awareness and Behaviors}

When compared with the correct answer rate of $30.0 \%$ for knowledge about fertility and age in a previous study [15], $95.0 \%$ and $86.7 \%$ of the subjects in this study had knowledge about oocyte aging and decrease of fertility with aging, respectively. In other words, the subjects in this study comprised a group with many people who were aware of these issues. In another previous study [26], 67.0\% and $86.6 \%$ of the subjects had no knowledge about underweight in pregnant women and low-birth-weight infants and no knowledge about low-birth-weight infants and lifestyle-related diseases, respectively. In this study, $66.7 \%$ and $86.7 \%$ of the subjects had no knowledge about underweight in pregnant women and low-birth-weight infants and no knowledge about low-birth-weight infants and lifestyle-related diseases, respectively, which were the same results as in the previous study. It was considered that communicating the effects of underweight on the fetus to unmarried women and women before pregnancy leads to the prevention of low-birth-weight infants. In a previous study [27], 87.5\% of subjects had no knowledge about folic acid and neural tube closure disorder in the fetus. In this study, $81.7 \%$ of the subjects had no knowledge about it. The Ministry of Health, Labour and Welfare of Japan has recommended folic acid intake for prevention of neural tube closure disorder in the fetus since 2000, but even after 18 years, around $80 \%$ of women of reproductive age answered "I don't know about it" when asked about it. For prevention of neural tube closure disorder in the fetus, intake of folic acid is necessary from 1 month before pregnancy to 3 months of pregnancy [23]. Therefore, it was considered important to educate women before pregnancy through PCC seminars and leaflets about the fact that folic acid is needed for prevention of neural tube closure disorder in the fetus. Regarding cervical cancer screening from age 20,78.3\% of the subjects answered "I know about it," and it is considered that awareness has increased 
due to educational activities such as the distribution of free vouchers for cervical cancer screening at 20 years of age.

As shown in Table 5, 93.3\% of the subjects answered that they prevent sexually transmitted diseases in the survey on PCC awareness and behaviors. However, as shown in Table 4, only $41.7 \%$ of the subjects knew about sexually transmitted diseases and infertility. Hence, it was considered that it was necessary to provide further education about the adverse effects caused by sexually transmitted diseases on infertility. As shown in Table 4, 78.3\% of the subjects knew about cervical cancer screening, but only $28.3 \%$ of the subjects actually undergo cervical cancer screening; therefore, it was thought that more support is needed for recommending consultations and examinations.

\subsection{Evaluation of PCC Health Education Program up to 6 Months after the Seminar}

\section{1) PCC knowledge}

As shown in Table 4, regarding the items of PCC knowledge other than oocyte aging and pregnancy/childbirth and life planning, the subjects acquired knowledge through this seminar, and the knowledge was maintained until 6 months after the seminar. Among these items, $100 \%$ of the subjects answered "I know about it" for rubella prevalence in pregnant women and the effects on the fetus at both 3 and 6 months after the seminar. For this item, a video showing a mother who suffered from rubella during pregnancy and lost her child was used in the seminar [25]. It was considered that use of the video succeeded in making an impression as an emotional experience in the Trans-theoretical Model [12], [13]. Regarding viewing of the video, "the video was impressive" was provided as a description in the impressions and free descriptions for the seminar and leaflets. Thus, it seems that viewing the video had an impact on maintaining knowledge.

\section{2) PCC awareness and behaviors}

As shown in Table 5, among the items of PCC awareness and behaviors, the values for actively eat foods containing folic acid, prevent sexually transmitted diseases, and conduct self-examinations for breast cancer were significantly increased at 3 months after the seminar. For actively eat foods containing folic acid, a change in awareness was seen along with that in knowledge. Breast cancer screening is not included in workplace health checkups for women in their twenties and thirties. To induce behavior change with respect to undergo breast cancer screening, behavior change was promoted through the seminar and feedback comments as helping relationships in the Trans-theoretical Model [12], [13]; however, it did not lead to the action of having the screening. On the other hand, for conduct self-examinations for breast cancer, it was considered that the explanation at the seminar with the demonstration led subjects to introduce the behavior into their daily lives and resulted in behavior change. Furthermore, the numbers of subjects who have been vaccinated against rubella, undergo medical examinations, and undergo breast cancer screening were decreased at 3 months 
after the seminar. This might have been due to it being unclear if the questions meant from the seminar until 3 months later or from before the seminar until 3 months later; therefore, it is necessary to revise the contents of the questionnaire.

\section{3) Nutrient intake}

As shown in Table 6, in the pre-seminar survey, the nutrients that were taken in proper amounts were energy, phosphorus, vitamin $\mathrm{K}$, and salt equivalent; the nutrients that were consumed a lot were protein, lipid, and carbohydrates; and the nutrients that were taken in insufficient amounts were calcium, magnesium, iron, vitamin D, vitamin C, and folic acid [28]. Regarding folic acid in particular, half of the subjects answered that they actively eat foods containing folic acid in the survey of PCC awareness and behaviors at 3 months after the seminar, but there was no increase in intake. Consumption of foods containing folic acid was encouraged through provision of the results for folic acid intake in the food frequency questionnaire as well as feedback comments as self-reevaluation in the Trans-theoretical Model [12] [13]; however, there was no change. Thus, it was considered that this was difficult to improve by only promoting food intake. The need for more proactive measures such as recommendation of use of supplements and processed foods with added folic acid (folic acid-enriched rice, folic acid-enriched eggs, folic acid-containing bread, folic acid-containing milk) and promotion of the addition of folic acid to cooking ingredients, which were described in a previous study [29], was suggested.

4) Health awareness and stress management of the Health-Promotion Lifestyle Profile scale and self-efficacy

As shown in Table 7, regarding health awareness and stress management, which are subscales of the Health-Promotion Lifestyle Profile scale, and self-efficacy, the value for health awareness was significantly increased at 6 months after the seminar. In a previous study [30], internal health locus of control was significantly increased at 2 weeks after a PCC intervention, and although the evaluation periods were different, 2 weeks and 6 months, similar results were obtained in this study. Another previous study [31] reported that there were no effective effects on self-efficacy, behavioral intent, and stress management in a survey 14 weeks after a PCC intervention. Similarly, there were no effective effects on self-efficacy and stress management in this study even at 6 months after the intervention.

\section{5) Group discussion}

A group discussion is an opportunity to promote behavior change by discussing obstacles to taking action based on empathy for how to perceive problems, and its effectiveness has been recognized in a previous study [31]. In this study, the group discussion was an opportunity to share correct knowledge within the group by asking questions raised during the seminar. In addition, listening to the opinions of the subjects was an opportunity to hear their questions specifically, such as "I didn't know what was wrong with not taking folic acid," which did not appear in the free descriptions after the seminar. These contents of the group discussion will be used when revising the leaflets or seminar. 


\section{6) Feedback comments}

Feedback comments were mailed within one week after the seminar as a reward, self-reevaluation in the Trans-theoretical Model [12] [13]. At 3 months after the seminar, awareness regarding actively eat foods containing folic acid was significantly increased. It was considered that awareness regarding actively eating foods containing folic acid could be enhanced not only the seminar but also by showing in feedback comments the amount of folic acid actually ingested. However, there was no change in the intake of folic acid, and there were also no significant changes in breast cancer screening, cervical cancer screening, and vaccination against rubella. Behavior change was only seen for self-examination for breast cancer. We believe that this was caused not only by the effects of the demonstration at the seminar but also by promotion through the feedback comments.

\subsection{Evaluation of the PCC Health Education Program by Participants}

In this evaluation, $81.7 \%$ of the participants in the PCC health education program indicated that the program is necessary, and the desired age at which they wanted to attend was in their early $20 \mathrm{~s}$. Because the subjects of this study were 20- to 35-year-old workers, it was difficult to secure the time for them to attend, and it seems that they wanted to be able to attend such a program in university, when they had enough time. Regarding the nutrient intake status report and physical activity results, $91.6 \%$ of the subjects indicated that they were useful. For short comments, $98.3 \%$ of the subjects indicated that they were useful. Many of the subjects said that the comments were more useful than the nutrient intake status report and physical activity results because it was difficult to read and understand the report and results, whereas they were able to understand the results because of the short comments. Regarding the free descriptions, the evaluations were generally positive, including such things as it was an opportunity to review my eating habits and it was an opportunity to think about lifestyle habits. On the other hand, there were also opinions pointing out issues, including such things as the food frequency questionnaire was difficult to complete and the nutrition results table was difficult to understand. Therefore, it will be necessary to revise the questionnaire to make it easier to answer and to make the results table easier to understand in the future.

Based on the above, study hypothesis 1 was verified because the percentage of subjects with PCC knowledge was significantly higher at 3 and 6 months after the health education seminar than before the seminar. For PCC awareness and behaviors, the percentages of subjects with awareness about preventing sexually transmitted diseases, conducting self-examinations for breast cancer, and actively eating foods containing folic acid were significantly increased; however, folic acid intake did not increase. This was partially verified because there was a significant increase in the percentage of respondents who eat well-balanced meals, make the mind active, prevent sexually transmitted diseases, and conduct self-examinations for breast cancer at 6 months after the PCC seminar. Regard- 
ing self-efficacy and the health awareness and stress management subscales of the Health-Promotion Lifestyle Profile scale, study hypothesis 2 was partially verified because there was a significant difference in the score for the health awareness subscale between before the health education seminar and 6 months after the seminar.

Based on the above, evaluation of this program revealed that: 1) the goal of female workers of reproductive age being able to learn about PCC was achieved; and 2) the goal of female workers of reproductive age being able to act based on PCC knowledge was partially achieved.

\subsection{Limitations of the Study and Challenges in the Future}

The limitations of this study include the fact that the percentage of highly educated subjects was relatively high because the chain sampling method was used and the fact that no control group was set. As a challenge in the future, it is necessary to enhance the accuracy of the results by increasing the number of subjects and conducting an intervention survey with a control group.

\section{Conclusions}

Evaluation of the health education program for PCC education in female workers of reproductive age up to 6 months after the seminar revealed the following:

1) The percentages of individuals with PCC knowledge at 3 and 6 months after the health education seminar were obviously higher than that before the seminar;

2) PCC knowledge was maintained for 6 months as a result of the seminar on PCC and intervention including the group discussion, food frequency questionnaire results, and feedback comments;

3) The percentage of individuals with PCC awareness and behaviors was increased for the items of actively eating foods containing folic acid, prevent sexually transmitted diseases, and conduct self-examinations for breast cancer at 3 months after the seminar and for the items of eat well-balanced meals, make the mind active, prevent sexually transmitted diseases, and conduct self-examinations for breast cancer at 6 months after the seminar;

4) Regarding the Health-Promotion Lifestyle Profile scale, the score for health awareness was significantly increased at 6 months after the seminar.

The present health education program established PCC knowledge and improved some of PCC awareness and behaviors after 6 months. Therefore, it was suggested that it was effective for PCC education for female workers of reproductive age.

\section{Acknowledgements}

The authors would like to express their sincere appreciation to all the participants who were willing to cooperate with this research as well as to everyone who referred the subjects to us. In addition, we would also like to express our deep appreciation to Professor Sasaki, Professor Tanaka, and Professor Dote for their guidance. 


\section{Conflicts of Interest}

The authors declare no conflicts of interest regarding the publication of this paper.

\section{References}

[1] Arata, N. (2016) Preconceptional Health Care and Postpartum Management in the View of Maternal Medicine. Journal of Clinical and Experimental Medicine, 256, 199-205. (In Japanese)

[2] Furuhashi, N., Kimura, H. and Nagae, H. (1992) Preconception Care. Obstetrical and Gynecological Practice, 41, 735-739. (In Japanese)

[3] Johnson, K., et al. (2006) Recommendation to Improve Preconception Health and Health Care-United States. A report of the CDC/ATSDR Preconception Care Work Group and the Select Panel on Preconception Care. MMWR Recommendations and Reports, 55, 1-23.

[4] Ministry of Education, Culture, Sports, Science and Technology Japan. Junior High School Study Guidance. (In Japanese)

http://www.mext.go.jp/component/a_menu/education/micro_detail/_icsFiles/afield $\underline{\text { file/2017/06/21/1384661_5_1.pdf }}$

[5] Ministry of Education, Culture, Sports, Science and Technology Japan. High School Study Guidelines. (In Japanese)

http://www.mext.go.jp/component/a_menu/education/micro_detail/_icsFiles/afield file/2011/03/30/1304427_003.pdf

[6] Health, Labour and Welfare Statistics Association (2018) Health and Welfare Statistics. Journal of Health and Welfare Statistics Japan, 65, 1021-1079. (In Japanese)

[7] NPO Japan Reproductive Health Association (2008) Manual for Infertility Prevention. Tokyo, Mothers' and Children's Health Organization Co., Ltd. (In Japanese)

[8] National Institute of Population and Social Security Research (2015) 15th Birth Trend Basic Survey Japan. (In Japanese)

http://www.ipss.go.jp/ps-doukou/j/doukou15/NFS15_gaiyou.pdf

[9] Barker, D.J.P., Osmond, C., Winter, P.D., et al. (1989) Weight in Infancy and Death from Ischaemic Heart Disease. The Lancet, 2, 577-580.

https://doi.org/10.1016/S0140-6736(89)90710-1

[10] Ministry of Health, Labour and Welfare Japan (2016) The Situation of Working Women. (In Japanese) http://www.mhlw.go.jp/bunya/koyoukintou/josei-jitsujo/dl/16b.pdf

[11] Mitsubishi Estate Co., Ltd. Luvtelli. (2016) Tertiary Stage Marunouchi Health Room Report. (In Japanese)

http://shokumaru.jp/willconscious_image/019_wp_info/book/wcm_wp2016/FLAS $\underline{\mathrm{H} / \mathrm{index} . \mathrm{Html}}$

[12] Prochaska, J.O., et al. (1995) Changing for Good: A Revolutionary Six-Stage Program for Overcoming Bad Habits and Moving Your Life Positively Forward.

[13] Prochaska, J.O., et al. (2005) Changing for Good. Houken Corp., Tokyo. (In Japanese)

[14] Arimoto, Y. and Saitho, M. (2010) Relationship between Unmarried Women's Knowledge of Reproduction and Their Life Plan. Journal of the Japan Maternal and Infant Care Association, 4, 13-21. (In Japanese)

[15] Yoshimura, Y. and Takahashi, K. (2015) Excel Eiyokun Food Frequency Questionnaire Based on Food Groups Ver4.0. Tokyo, Kenpakusha. (In Japanese) 
[16] Nagusa, M. (2019) Literature Examination about the Pre-Conception Care of Maturation Period Women. Journal of Japanese Society of Human Caring Research, 10, 9-17. (In Japanese)

[17] Sherer, M., Waddux, J.E., Wercandante, B., Printice-Dunn, S., Jacobs, B. and Rogers, R.W. (1982) The Self-Efficacy Scale: Construction and Validation. Psychological Reports, 51, 663-671. https://doi.org/10.2466/pr0.1982.51.2.663

[18] Hori, H. and Yamamoto, M. (2012) Collection of Psychometrics Standards I We Feel the Human inside < the Individual, Process in Self $>$. SAIENSU-SHA Co., Ltd., Tokyo, 37-41. (In Japanese)

[19] Walker, S.N., Sechrist, K.R. and Pender, N.J. (1987) The Health-Promoting Life-Style Profile: Development and Psychometric Characteristics. Nursing Research, 36, 76-81. https://doi.org/10.1097/00006199-198703000-00002

[20] Wei, C., Yonemitsu, H., Harada, K., et al. (1994) A Japanese Language Version of the Health-Promoting Lifestyle Profile. Japanese Journal of Public Health, 54, 597-606. (In Japanese) https://doi.org/10.1265/jjh.54.597

[21] Sato, Y. (2017) Pre-Conception Care to Begin on Today. Withmedical Co., Ltd., Gunma. (In Japanese)

[22] NHK Press Pool (2013) Shock of the Ovum Aging Not to Be Able to Produce though We Want to Give Birth. Bungeishunju Ltd., Tokyo. (In Japanese)

[23] Ministry of Health, Labour and Welfare Japan (2000) About Promotion of the Appropriate Reporting to Modify the Folic Acid Intake for Women with Childbearing Age for Onset Risk Reduction of the Medullary Tube Choke Disorder. http://www.mhlw.go.jp/houdou/2006/02/dl/h0201-3a3-03c.pdf

[24] Ministry of Health, Labour and Welfare Japan (2014) About Rubella. http://www.mhlw.go.jp/seisakunitsuite/bunya/kenkou_iryou/kenkou/kekkaku-kans enshou/rubella/index.Html

[25] Sato, A. (2013) Stop Rubella-Do You Know Congenital Rubella Syndrome. http://www.change.org/fushin

[26] Mikamo, S. and Machiura, M. (2013) Eating Habits, Knowledge Regarding Leanness and Personal Values among Young, Pre-Pregnant, Working Females in Their 20s and 30s-A Comparison of Females in Underweight and Normal Weight Groups. Japanese Journal of Maternal Health, 53, 522-529. (In Japanese)

[27] Mikamo, S. and Machiura, M. (2012) Comparison between Knowledge and General Office Work before the Actual Situation Pregnancy of Eating Habits about Effect and Folic Acid of Emaciation of the Nurse before the Pregnancy. Japanese Nursing Society Memoirs: Maternal Nursing, 42, 92-95. (In Japanese)

[28] Hishida, A. and Sasaki, S. (2014) Dierary Reference Intakes for Japanese, 2015. DAI-ICHI SHUPPAN Co., Ltd., Tokyo. (In Japanese)

[29] Sato, Y., Nakanishi, T., Chiba, T., et al. (2014) Attitudes of Pregnant Japanese Women and Folic Acid Intake for the Prevention of Neural Tube Defects: A Nationwide Internet Survey. Japanese Society of Public Health, 61, 321-332. (In Japanese)

[30] Bastani, F., Hashemi, S., Bastani, N., et al. (2010) Impact of Preconception Health Education on Health Locus of Control and Self-Efficacy in Women. Eastern Mediterranean Health Journal, 16, 396-401. https://doi.org/10.26719/2010.16.4.396

[31] Hillemeier, M.M., Downs, D.S., Feinberg, M.E., et al. (2008) Improving Women's Preconceptional Health: Findings from a Randomized Trial of the Strong Healthy Women Intervention in the Central Pennsylvania Women's Health Study. Women's Health, 18, 87-96. https://doi.org/10.1016/j.whi.2008.07.008 